\title{
RADIO OBSERVATIONS OF GRB 100418a: TEST OF AN ENERGY INJECTION MODEL EXPLAINING LONG-LASTING GRB AFTERGLOWS
}

\author{
A. Moin ${ }^{1,2}$, P. Chandra ${ }^{3}$, J. C. A. Miller-Jones 2 , S. J. TingaY ${ }^{2}$, G. B. TaYlor ${ }^{4,5}$, D. A. Frail ${ }^{5}$, \\ Z. WAng ${ }^{1}$, C. ReYnolds ${ }^{2}$, ANd C. J. Phillips 6 \\ ${ }^{1}$ Shanghai Astronomical Observatory, 80 Nandan Road, Xujiahui, Shanghai 200030, China \\ 2 International Centre for Radio Astronomy Research, Curtin University, Bentley 6102, WA, Australia \\ ${ }^{3}$ National Center for Radio Astrophysics, Tata Institute of Fundamental Research, Pune, India \\ ${ }^{4}$ Department of Physics and Astronomy, University of New Mexico, Albuquerque, NM 87131, USA \\ ${ }^{5}$ National Radio Astronomy Observatory, P.O. Box 0, Socorro, NM 87801, USA \\ ${ }^{6}$ CSIRO Astronomy and Space Science, PO Box 76, Epping, NSW, Australia \\ Received 2013 July 5; accepted 2013 October 8; published 2013 November 27
}

\begin{abstract}
We present the results of our radio observational campaign of gamma-ray burst (GRB) 100418a, for which we used the Australia Telescope Compact Array, the Very Large Array, and the Very Long Baseline Array. GRB 100418a was a peculiar GRB with unusual X-ray and optical afterglow profiles featuring a plateau phase with a very shallow rise. This observed plateau phase was believed to be due to a continued energy injection mechanism that powered the forward shock, giving rise to an unusual and long-lasting afterglow. The radio afterglow of GRB 100418a was detectable several weeks after the prompt emission. We conducted long-term monitoring observations of the afterglow and attempted to test the energy injection model advocating that the continuous energy injection is due to shells of material moving at a wide range of Lorentz factors. We obtained an upper limit of $\gamma<7$ for the expansion rate of the GRB 100418a radio afterglow, indicating that the range-of-Lorentz factor model could only be applicable for relatively slow-moving ejecta. A preferred explanation could be that continued activity of the central engine may have powered the long-lasting afterglow.
\end{abstract}

Key words: gamma-ray burst: individual (GRB 100418a) - radiation mechanisms: non-thermal - radio continuum: general - relativistic processes - stars: winds, outflows

\section{INTRODUCTION}

Gamma-ray burst (GRB) 100418a was detected by the Swift satellite at 21:10:08 UT, 2010 April 18. The Burst Alert Telescope (BAT) on board the Swift satellite was triggered by the GRB and after the initial trigger, Swift's X-Ray Telescope (XRT) and Ultraviolet/Optical Telescope (UVOT) slewed to the source and detected it in the X-ray and optical bands (Marshall et al. 2011). Subsequently, a number of ground-based telescopes carried out detailed studies of the afterglow of GRB 100418a. Optical observations with the Very Large Telescope (VLT) and the Gemini North Telescope determined the redshift of GRB 100418a to be 0.6235 (Antonelli et al. 2010; Cucchiara \& Fox 2010).

Marshall et al. (2011) analyzed the Swift BAT, XRT, and UVOT data and reported that the afterglow of the GRB exhibited unusual behavior in the optical and X-ray bands. After a brief period of steep decline, the X-ray light curve featured an unusually long "plateau" phase, lasting from about $600 \mathrm{~s}$ until 50-90 ks after the burst. The optical afterglow also showed a period of re-brightening with a similar power-law index, from about $87 \mathrm{~s}$ until $51 \mathrm{ks}$ after the burst. Following this shallow rise in the light curves, the afterglow resumed a steeper and more normal power-law decay.

Jia et al. (2012) and Niino et al. (2012) independently found that there was no evidence of a supernova association with GRB 100418a. Together with the high metallicity of the host galaxy (de Ugarte Postigo et al. 2011; Niino et al. 2012), the absence of a supernova and a peculiar, long-lasting afterglow leads to the inference that either the progenitor belongs to an entirely different class or that it behaved in a way different from how the GRB afterglow would be expected to behave within the framework of the standard collapsar-fireball model (MacFadyen \& Woosley 1999; MacFadyen et al. 2001).
Long-term, multi-wavelength observational studies of the afterglow of peculiar GRBs such as GRB 100418a provide an opportunity to examine the unusual behavior of afterglows of these rare GRBs, test previously advanced theories of GRB physics (Rees \& Meszaros 1992, 1998; Panaitescu et al. 1998; Waxman et al. 1998; Dai \& Lu 1998; Zhang et al. 2006), and hold the potential to find clues to the nature of GRB central engines. Motivated by the unusual afterglow behavior, we conducted radio monitoring of GRB 100418a in an attempt to understand the astrophysical mechanism behind the afterglow profile and the role of the central engine and to test the "energy injection" model (Rees \& Meszaros 1998; Zhang et al. 2006), which offers a plausible explanation of the physics of long-term afterglows of GRBs. While a long-lasting afterglow could in principle be explained by the jets running into the dense circumburst medium (with the time delay between approaching and receding jets prolonging the afterglow), the lack of an associated supernova led us to focus this work on the energy injection model. In what follows, we show that the unique behavior of the afterglow of this GRB supplies some observational evidence to support one of the postulates of the energy-injection model, which advocates continued activity of the central engine as the primary driver for long-lasting afterglows.

\section{OBSERVATIONS AND RESULTS}

Radio monitoring of GRB 100418a was conducted using the Australia Telescope Compact Array (ATCA) in Australia (Moin et al. 2010) and the Very Large Array (VLA) in the United States (Chandra \& Frail 2010). Owing to the significant and consistent brightness at radio wavelengths, observations were conducted with the Very Long Baseline Array (VLBA) to observe the GRB afterglow at high angular resolution. Figure 1 shows the combined VLA + ATCA light curve for the radio afterglow of GRB 100418a. It also includes the VLBA observations. 


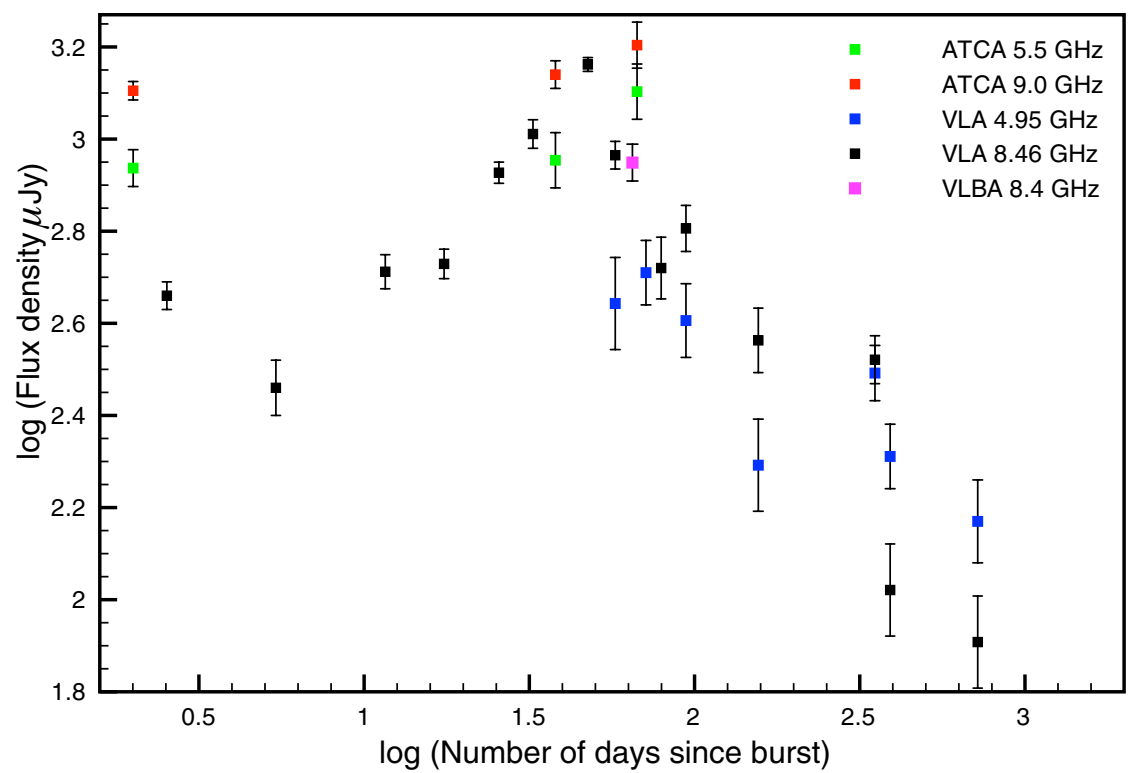

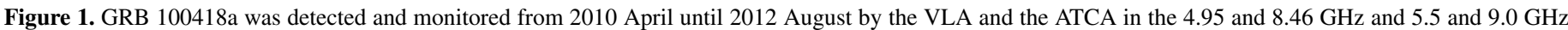

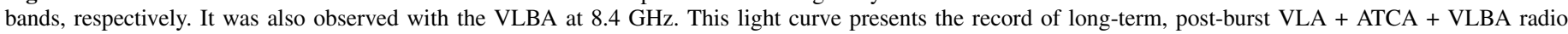
observations (error bars: $1 \sigma$ ) of the GRB 100418a afterglow.

\subsection{VLA Observations}

VLA observations of GRB 100418a started 2010 April 21. Until 2010 September 9, the observations were conducted at 4.95 and $8.46 \mathrm{GHz}$. Subsequently, observations were carried out at 4.9 and $7.9 \mathrm{GHz}$. The VLA monitoring continued through 2012 August. The data were taken in the interferometric mode for an average of 30 minutes (including calibrator time). 3C286 was used as the flux calibrator as well as the bandpass calibrator. $\mathrm{J} 1728+1215$ was used for the phase calibration. The data were analyzed using standard AIPS $^{7}$ (Greisen 2003) routines. The flux density of the radio afterglow continued to rise until it reached a peak about 60 days after the prompt emission and the post-peak phase featured a gradual decline as the radio afterglow was fading away. Details of all the radio observations are listed in Table 1.

\subsection{ATCA Observations}

GRB 100418a was observed using the ATCA during three epochs of an ongoing GRB observation and monitoring program (ATCA proposal C1802; Moin 2012), conducted in 2010 April, May, and June (see Table 1). It was detected at all three epochs at 5.5 and $9.0 \mathrm{GHz}$.

The source was observed with a nearby calibrator (J1655+077) for phase referencing and PKS 1934-638 was observed during the session for primary flux and bandpass calibration. Data were taken with the Compact Array Broadband Backend (Wilson et al. 2011) in the standard continuum mode with a bandwidth of $2.048 \mathrm{GHz}$, in two polarizations.

The output data files were then loaded into MIRIAD ${ }^{8}$ (Sault et al. 1995) for calibration. Standard MIRIAD calibration routines were used to calibrate the amplitude and the phase gains of the data and FITS files of the calibrated data were produced, which were then loaded into Difmap ${ }^{9}$ (Shepherd 1997) to produce final images of the GRB 100418a afterglow. In each image, the detected afterglow was fit with a point-source

\footnotetext{
7 http://www.aips.nrao.edu/index.shtml

8 http://www.atnf.csiro.au/computing/software/miriad/

9 ftp://ftp.astro.caltech.edu/pub/difmap/difmap.html
}

model using the model-fitting routine with five iterations and the peak flux density for each session was determined. The errors on flux densities are the $1 \sigma$ value of the rms noise.

Even a number of weeks after the burst, it was possible to detect the radio afterglow, as can be seen from Figure 1, which was quite unusual as the likelihood of detecting the radio afterglow of most GRBs starts to diminish within a few days of the prompt emission. The recent GRB radio afterglow catalog (Chandra \& Frail 2012) reported that the GRBs with radio afterglows lasting more than seven days comprised approximately $22 \%$ of the entire sample of GRBs observed as part of the GRB radio follow-up campaign running since 1997 and the GRB radio afterglows that were monitored and studied over several months comprised only approximately $5 \%$ of the full sample.

The ATCA + VLA light curve features a brief decline before it starts to rise from day 11 onward until it reached its peak about 60 days after the burst. There were no further ATCA observations of the GRB 100418a radio afterglow after the third session in 2010 June but the VLA continued to monitor it. The spectral indices from the late-time observations of the GRB do indicate that the afterglow emission transitioned from an inverted to a steep spectrum, which is an expected feature of long-lasting radio afterglows at late times. Noticeable short timescale variations between the ATCA flux densities and those obtained from the VLA epochs are possibly due to the diffractive scintillation effects in the ionized interstellar medium (Frail et al. 2000), with additional contributions from the differences in the flux and phase calibrators that were used at both observatories to calibrate the GRB 100418a data.

\subsection{VLBA Observations}

The very long baseline interferometry (VLBI) observations of GRB 100418a were conducted in an attempt to resolve the afterglow and to determine the possible milliarcsecond (mas)scale structure associated with the afterglow. Previous VLBI monitoring of the bright and nearby GRB 030329 provided important clues to the evolution and behavior of GRB radio afterglows (Taylor et al. 2004, 2005; Pihlström et al. 2007). 
Table 1

VLA, ATCA, and VLBA Observations of GRB 100418a

\begin{tabular}{|c|c|c|c|c|c|c|}
\hline Date & Instrument & $\begin{array}{c}\delta T \\
\text { (days) }\end{array}$ & $\begin{array}{l}\text { Frequency } \\
\quad(\mathrm{GHz})\end{array}$ & Configuration & $\begin{array}{l}\text { Flux Density } \\
\left(\mu \mathrm{Jy} \mathrm{beam}^{-1}\right)\end{array}$ & $\begin{array}{c}\mathrm{rms} \\
\left(\mu \mathrm{Jy} \mathrm{beam}^{-1}\right)\end{array}$ \\
\hline 2010 Apr 20 & ATCA & 2 & 5.5 & $6 \mathrm{~A}$ & 865 & 120 \\
\hline 2010 Apr 20 & ATCA & 2 & 9.0 & $6 \mathrm{~A}$ & 1273 & 96 \\
\hline 2010 Apr 21 & VLA & 3 & 8.46 & $\mathrm{D}$ & 458 & 20 \\
\hline 2010 Apr 24 & VLA & 6 & 8.46 & $\mathrm{D}$ & 289 & 22 \\
\hline 2010 Apr 30 & VLA & 12 & 8.46 & $\mathrm{D}$ & 516 & 22 \\
\hline 2010 May 6 & VLA & 18 & 8.46 & $\mathrm{D}$ & 537 & 20 \\
\hline 2010 May 14 & VLA & 26 & 8.46 & $\mathrm{D}$ & 847 & 23 \\
\hline 2010 May 21 & VLA & 33 & 8.46 & $\mathrm{D}$ & 1028 & 37 \\
\hline 2010 May 26 & ATCA & 38 & 5.5 & $6 \mathrm{C}$ & 900 & 80 \\
\hline 2010 May 26 & ATCA & 38 & 9.0 & $6 \mathrm{C}$ & 1370 & 180 \\
\hline 2010 Jun 5 & VLA & 48 & 8.46 & $\mathrm{D}$ & 1454 & 21 \\
\hline 2010 Jun 15 & VLA & 56 & 4.95 & $\mathrm{D}$ & 440 & 76 \\
\hline 2010 Jun 15 & VLA & 56 & 8.46 & $\mathrm{D}$ & 923 & 32 \\
\hline 2010 Jun 22 & VLBA & 65 & 8.4 & & 890 & 50 \\
\hline 2010 Jun 26 & ATCA & 67 & 5.5 & $6 \mathrm{C}$ & 1270 & 120 \\
\hline 2010 Jun 26 & ATCA & 67 & 9.0 & $6 \mathrm{C}$ & 1600 & 200 \\
\hline 2010 Jun 29 & VLA & 72 & 4.95 & $\mathrm{D}$ & 513 & 44 \\
\hline $2010 \mathrm{Jul} 7$ & VLA & 80 & 8.46 & D & 526 & 41 \\
\hline 2010 Jul 22 & VLA & 95 & 8.46 & $\mathrm{D}$ & 641 & 37 \\
\hline 2010 Jul 22 & VLA & 95 & 4.95 & $\mathrm{D}$ & 404 & 50 \\
\hline 2010 Sep 22 & VLA & 157 & 8.46 & DnC & 366 & 53 \\
\hline 2010 Sep 22 & VLA & 157 & 4.95 & DnC & 196 & 64 \\
\hline 2011 Apr 6 & VLA & 353 & 4.9 & B & 311 & 25 \\
\hline 2011 Apr 6 & VLA & 353 & 7.9 & B & 332 & 20 \\
\hline 2011 Sep 15 & VLA & 391 & 4.9 & A-D & 205 & 82 \\
\hline 2011 Sep 15 & VLA & 391 & 7.9 & A-D & 105 & 26 \\
\hline 2012 Aug 21 & VLA & 721 & 4.9 & B & 148 & 28 \\
\hline 2012 Aug 21 & VLA & 721 & 7.9 & B & 81 & 34 \\
\hline
\end{tabular}

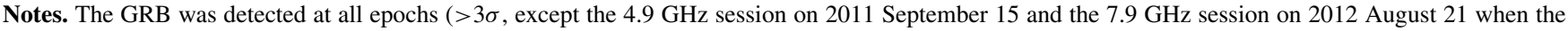
detections were $<3 \sigma)$. $\delta \mathrm{T}$ is the number of the days since prompt emission.

VLBA observations of GRB 100418a were carried out as part of program BM347 on 2010 June 22. The source was observed for eight hours with full Stokes parameters (dual polarization) at a frequency of $8.4 \mathrm{GHz}$ with a bandwidth of $8 \times 8 \mathrm{MHz}$. Along with the target source, the phase-reference calibrator $\mathrm{J} 1706+1208$ and the VLBA fringe finder 3C345 were also observed. The data were correlated with the DiFX correlator in Socorro (Deller et al. 2007, 2011).

The correlated data were reduced and processed in AIPS (Greisen 2003) as a first step to performing phase and amplitude calibration. Single-source FITS files containing calibrated data were produced and then loaded into Difmap for preliminary model fitting and imaging. A cell size of $0.1 \times 0.1$ mas was used and the image was produced with natural weighting. The outcome of the VLBI observations and data analysis revealed an unresolved radio source associated with GRB 100418a having a correlated flux density of $890 \pm 50 \mu \mathrm{Jy}$, where the error is the $1 \sigma$ rms noise.

Figure 2 is the VLBI image of the radio afterglow of GRB $100418 \mathrm{a}$ at $8.4 \mathrm{GHz}$. An estimate of its angular size and expansion rate could help constrain the energy injection model. It is interesting to note that the VLBA flux density matches almost exactly with the flux density obtained from the VLA observation on the same day, indicating that there was no missing flux due to the long baselines of the VLBA resolving out any structure in the afterglow.

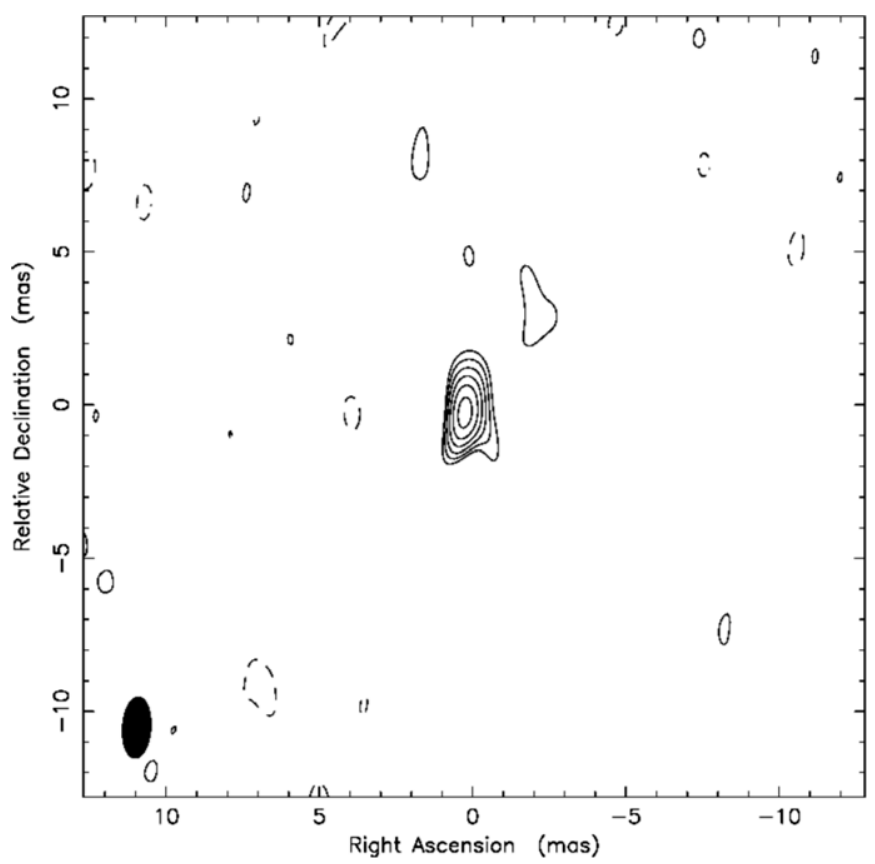

Figure 2. GRB 100418a VLBI image at $8.4 \mathrm{GHz}$ (2010 June 22). Contour levels: $-10 \%, 10 \%, 20 \%, 40 \%$, and $80 \%$ of the peak intensity. The peak flux density is $890 \pm 50 \mu \mathrm{Jy}(1 \sigma)$, the beam size is $1.99 \times 0.919$ mas, and the position angle is -3.7 . 
The GRB 100418a radio afterglow position obtained from the VLBA observations is 17:05:27.092 $\pm 0.005,+11: 27: 42.24 \pm$ 0.01 (calibrator position: 17:06:20.4974, +12:08:59.794 with an uncertainty of 0.5 mas; http://www.astrogeo.org). The errors were estimated on the basis of the beam size and signal-to-noise ratio considerations. A systematic uncertainty of 0.06 mas was estimated in both coordinates based on the calibrator-target separation (which was 0.72 in this case; Pradel et al. 2006). The VLBI position is consistent with the position of the optical counterpart detected by GROND with an error circle of 0.5 arcsec (Klose 2010), as well as the position of the radio counterpart detected by the ATCA and VLA.

The source was unresolved at the VLBA beam size of $1.99 \times$ 0.919 mas. An upper limit on the angular extent of the source of $<0.33$ mas $(1 \sigma)$ was obtained by performing model fitting in both the $(u, v)$ and image planes using elliptical Gaussian models in AIPS. The upper limit ruled out that there was any larger scale structure at flux densities above the detection threshold at the time of the VLBA observations of the afterglow at $8.4 \mathrm{GHz}$. Based on the size upper limit, the maximum physical size of $<1.375 \times 10^{18} \mathrm{~cm}$ and the apparent expansion speed $<8 \mathrm{c}$ were also determined. The VLBI observations of GRB 100418a were conducted about 65 days after the prompt emission and the source was found to be unresolved, so the derived upper limit on the apparent expansion speed was a long-term average assuming uniform expansion (mildly relativistic) and it does not conflict with possible highly relativistic expansion at early times.

The lower limit on the brightness temperature of the radio emission region was estimated as $T_{b}>2.95 \times 10^{9} \mathrm{~K}$. The value was determined following Taylor et al. (1999). This lower limit on the brightness temperature is high enough $\left(>10^{7} \mathrm{~K}\right)$ that the emission must be non-thermal. Therefore, the most likely emission mechanism is synchrotron emission resulting from the excitation of electrons during the interaction of the forward shock with the circumburst medium. Taking advantage of multifrequency observations, the spectral index $\alpha$ (where $S_{v} \propto v^{\alpha}$ ) from the ATCA epoch closest to the VLBI observations was estimated as $\alpha=0.5 \pm 0.3$, which indicates that the GRB radio afterglow was optically thick at $8.4 \mathrm{GHz}$.

\section{ANALYSIS AND INTERPRETATION}

GRB 100418a was ordinary in terms of the estimated energy release and the luminosity, but what made it an extraordinary GRB was the unusual afterglow emission behavior, i.e., the plateau phase in X-ray, re-brightening in the optical, and the latetime rise and longevity of the radio afterglow. Zhang et al. (2006) proposed a model called the "energy injection model" to explain this kind of behavior. This model was invoked by Marshall et al. (2011) to answer questions concerning the mechanisms that could explain the unusual behavior of the GRB 100418a afterglow.

The radius of the radiating GRB shell can be crudely approximated as $R \sim \delta t c$, where $\delta t$ is the time between the prompt emission and the peak of the radio afterglow and $c$ is the speed of light (Katz 1994; Katz \& Piran 1997; Frail et al. 1997). From the VLA light curve, $R$ is estimated to be $\sim 1.56 \times$ $10^{17} \mathrm{~cm}$. This value obtained using this approximation is consistent with the predictions of the relativistic fireball model for GRB radio afterglows presented by Waxman (1997a, 1997b, 1997c). This model states that the radio emission associated with a GRB comes from a cone of the fireball along the observer's line of sight and $R$ is therefore the apparent radius of the cone, which is suggested to be the emitting region. The brief decline and then the significant rise in the radio afterglow light curve (Figure 1) is indicative of the fact that there must be some sort of energy injection mechanism that refreshed the forward shock and in turn re-energized the fading afterglow.

\subsection{Theoretical Framework}

Before attempting to explain the behavior of the GRB 100418a afterglow in the context of the energy injection model, it is important to put the observational signatures of the GRB 100418a afterglow in the context of the overall GRB phenomenon and the standard blast wave model (Rees \& Meszaros 1992; Meszaros \& Rees 1993, 1997a; Waxman 1997a; Wijers et al. 1997), which is still the most plausible physical framework, since the background physics proposed by this model has been repeatedly found to be in good agreement with GRB observations. The blast wave, or cosmological fireball model, proposes that a compact central engine produced as a result of the massive explosion of the progenitor star launches a relativistic outflow, releasing an enormous amount of energy (of the order of $10^{52} \mathrm{erg}$ ). The outflow powers internal shocks (Rees \& Meszaros 1994) with different Lorentz factors. Collisions between these internal shocks are thought to produce the GRB prompt emission. Following the internal shocks, a blast wave, also known as a forward shock or external shock, powered by the expanding ejecta, is driven into the circumburst medium, which is believed to produce the afterglow that is then observed at lower frequencies. The forward shock accelerates the electrons in the circumburst medium to relativistic speeds and they emit synchrotron radiation as they move in the surrounding magnetic field, which can then be detected and monitored for long-term afterglow studies (Meszaros \& Rees 1997b; Kulkarni et al. 1998; Sari et al. 1998; Waxman et al. 1998; Frail et al. 2000).

Studies have indicated that the various GRB afterglow phases seen in the light curves represent the transitions that the decelerating ejecta and the expanding blast wave might be going through. In other words, the variations in the afterglow light curves can help infer the state of the emitting region. Figure 1 of Zhang et al. (2006) shows the five possible components that the light curve of a GRB X-ray afterglow can have based on its decay index $\alpha\left(F_{v} \propto t^{-\alpha}\right)$. Marshall et al. (2011) showed that the X-ray light curve of GRB 100418 a exhibits three distinct phases: (1) a steep decline $(\alpha=4.18)$, (2) a shallow plateau phase $(\alpha=-0.23)$, and (3) a relatively normal decay $(\alpha=1.37)$. Phases $a$ and $c$ match well with segments I and III of Figure 1 of Zhang et al. (2006), respectively. However, the second phase of GRB 100418a X-ray afterglow features a very shallow rise instead of a shallow decline phase that matches with segment II of the Zhang et al. (2006) synthetic light curve.

The rapid early decay of the GRB 100418a light curves is common among most the GRBs and is attributed to the "GRB tail-emission," that is, the steeply declining prompt emission that comes from the internal shocks immediately after the burst (Dermer 2004; Liang et al. 2006; Zhang et al. 2006). In general, the transition from declining prompt emission to early X-ray emission manifests itself as the transition from a sharp decline to a shallow decline in the afterglow light curve (Tagliaferri et al. 2005).

The second component in the optical and X-ray light curves is the unusual plateau phase with a very shallow rise. This is a rare feature in terms of the duration and behavior and it appears to be a manifestation of some sort of a continuous post-burst activity. This behavior has only been seen in very few GRB X-ray afterglows (e.g., GRB 060729; Grupe et al. 2007). 
The energy injection model proposes that if the external (forward) shock that produces the GRB afterglow is continuously fed with energy after the initial supply, the forward shock keeps getting refreshed to produce a multi-wavelength afterglow for a longer period of time. According to the model, the onset of this continuous energy injection manifests itself as the very shallow plateau phase in the afterglow light curve, which is what is seen in the case of the afterglow of GRB 100418a.

The total isotropic kinetic energy in the case of GRB 100418a, which powered the plateau phase in the X-ray afterglow, was estimated by Marshall et al. (2011) to be $E_{k, \text { iso, } f} \geqslant 10^{53} \mathrm{erg}$, given $t_{i} \sim 600 \mathrm{~s}$ and $t_{p} \sim 50-90 \mathrm{ks}$, where $t_{i}$ is the time when the plateau phase starts and $t_{p}$ is the time when the afterglow reached its peak before the transition to a steep decay phase. This energy is 100 times the initial isotropic energy $\left(E_{k, \text { iso }, i} \sim 10^{51}\right.$; Marshall et al. 2011) and is thought to be continuously injected into the forward shock, which then produced a long-term, slowly varying afterglow (Dai \& Lu 1998; Zhang \& Meszaros 2001; Zhang et al. 2006). The energy budget is thus consistent with the energy injection model and plausibly explains the behavior exhibited by the afterglow of GRB 100418a.

The following mechanisms can give possible explanations for the energy injection model.

1. Ejecta with a wide range of Lorentz factors, transporting energy to the forward shock (Rees \& Meszaros 1998; Zhang et al. 2006).

2. Continued activity of the central engine producing a Poynting flux-dominated flow (Dai \& Lu 1998; Zhang et al. 2006).

In the first scenario, multiple spherical shells are ejected with a range of Lorentz factors. They reach the forward shock at different times, continuously refreshing the forward shock to give rise to a longer-lasting and slowly varying afterglow (Rees \& Meszaros 1998). This model can be described as a relation between the fractional mass $M_{\text {ej }}$ ejected with Lorentz factors above a certain value of $\gamma$ and the range of Lorentz factors of the shells of material:

$$
M_{\mathrm{ej}}(>\gamma) \propto \gamma^{-s}
$$

where $s$ is the mass outflow index and $\gamma$ is the Lorentz factor.

\subsection{Observational Constraints}

The GRB 100418a VLBI observations led us to test this model by determining an upper limit on the long-term average of the Lorentz factor of the ejecta $\gamma<7$, which we were able to obtain by estimating the upper limit on the apparent expansion speed as $<8 c$. The upper limit on the expansion speed was determined using the upper limit on the source size obtained from the VLBA data and estimating the value of angular diameter distance assuming a $\Lambda$ cosmology with $H_{0}=71 \mathrm{~km} \mathrm{~s}^{-1} \mathrm{Mpc}^{-1}, \Omega_{M}=0.27$, and $\Omega_{\Lambda}=0.73$ (Raine \& Thomas 2001; Taylor et al. 2004), at the redshift $(z=0.6235)$ of GRB 100418a. Using the formulation (Sedov-von NeumannTaylor solutions) presented in Frail et al. (2000), the equivalent isotropic energy $E_{0}$ associated with the radio afterglow can be given as

$$
E_{0} \approx 4.4 \times 10^{50} \eta_{1}^{-2 / 17} v_{*}^{-0.56} a_{*}^{35 / 17} b_{*}^{15 / 17} d_{*}^{70 / 17} \mathrm{erg},
$$

where $a_{*}, b_{*}, d_{*}, \eta_{1}$, and $v_{*}$ are the parameters that define the afterglow model developed by Frail et al. (2000) and are related to the fireball expanding at a subrelativistic velocity. Given the constraints (i.e., the upper limits on physical size and Lorentz factor) obtained from the VLBI observations, the values of the parameters in Equation (2) were calculated using the expressions given in the appendix of Frail et al. (2000). Those values were then used in Equation (2) and the upper limit on the value of the afterglow (fireball) energy is estimated to be $E_{0} \leqslant 5.8 \times$ $10^{51} \mathrm{erg}$. This value is two orders of magnitude less than the value of the isotropic kinetic energy estimated by Marshall et al. (2011) because the afterglow was in the radio regime more than eight weeks after the prompt emission and the accelerated particles had lost some energy, becoming less and less relativistic as the fireball was expanding in the circumburst medium.

The ejected mass $M_{\text {ej }}$ corresponding to the radio afterglow energy release can be estimated using the formulation presented in Panaitescu \& Kumar (2002), based on Einstein's equation relating relativistic mass and energy:

$$
M_{\mathrm{ej}} \approx \frac{E_{0}}{c^{2} \gamma_{0}} \mathrm{~kg}
$$

The upper limit on the value of mass corresponding to this energy release was determined to be $M_{\mathrm{ej}} \leqslant 0.46 \times 10^{-3} M_{\odot}$, where $M_{\odot}$ is the solar mass.

\subsection{Test of the Energy Injection Model}

The constraints on $\gamma$ and $M_{\mathrm{ej}}$ obtained from the VLBA observations allowed us to test the model relating the ejected mass and Lorentz factor (Equation (1)) against the observations from which it can be concluded that in case of GRB 100418a, the range-of-Lorentz-factor postulate of the energy injection model is in fact only valid for a small range of Lorentz factors, i.e., $\gamma<7$, and that 65 days after the GRB 100418a prompt emission, the fractional mass outflow and the energy release were indeed dominated by slow-moving ejecta with values of $\gamma<7$. That is, it is only the slow-moving shells that contribute to the longevity of the afterglow and there must have been another mechanism, such as extended activity of the central engine, which was driving the continued supply of energy to the forward shock in the beginning and in turn powering the long-lasting afterglow. The VLBA Lorentz factor limit $(\gamma<7)$ is model independent since it is a direct observation. Figure 3 shows the plots of Equation (1) for a range of mass outflow indices $0<s \leqslant 6$. The mass outflow is dominated by slowmoving shells for $s>1$ (Rees \& Meszaros 1998) and for $s>6$, the curves start to converge indistinguishably. It, therefore, is only a small window provided by the limits on $\gamma$ and $M_{\mathrm{ej}}$ within which the observations are consistent with the wide range of the Lorentz factor model (Rees \& Meszaros 1998; Zhang et al. 2006) and the model does not hold for higher values of $\gamma$. Our conclusion thus strengthens the argument in favor of the continued activity of the central engine as the process that primarily powers the prolonged afterglow.

In the case of continued activity of the central engine, the existing theoretical framework suggests that there could be at least some long-duration GRBs that are produced when a massive progenitor collapses into a neutron star or a highly magnetized pulsar (the proto-magnetar model; e.g., Metzger et al. 2011). During the birth of a pulsar or magnetar, the relativistic outflow produces internal shocks closer to the central engine that could produce the prompt emission, with the energy transported to a distance feeding the external shocks that produce the GRB afterglow. The central engine may then 


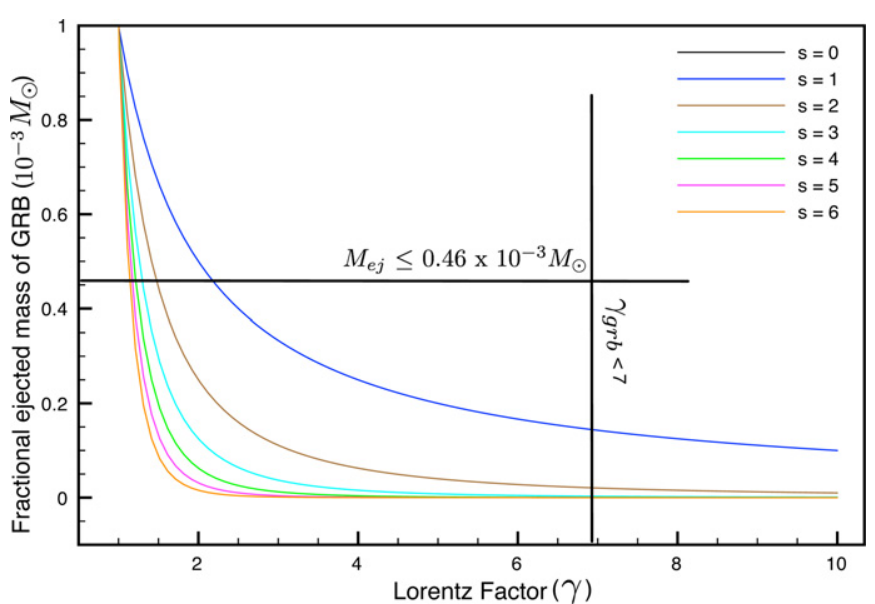

Figure 3. Relationship between the ejected GRB mass $M_{\mathrm{ej}}$ and Lorentz factor $\gamma$ (Equation (1)) plotted for different values of mass outflow index $s$ (Rees \& Meszaros 1998; Zhang et al. 2006). The estimation of upper limits on $M_{\mathrm{ej}}$ and $\gamma$ indicates that it is only within the window shown in the figure that the rangeof-Lorentz-factor hypothesis for energy injection may be true. That is, only for slow-moving ejecta with lower values of $\gamma$.

start to spin down and lose its energy by magnetic dipole radiation. In the presence of a strong electromagnetic field, the energy is transported via a relativistic magnetohydrodynamic wind as Poynting flux energy that keeps refreshing the external (forward) shock. As a result, the shock decelerates less slowly in the circumburst medium, resulting in continued emission of afterglow radiation (Duncan \& Thompson 1992; Thompson 1994; Zhang \& Meszaros 2001; Zhang et al. 2006).

Another possible scenario describes the continuous latetime accretion of "fall-back" material onto a black hole as the primary mechanism powering energy injection. It has been proposed that late-time hyper-accretion is sustained by distant chunks of material that eventually end up in the vicinity of the accreting black hole. This material is accreted onto the black hole, refreshing the accretion and fueling the long-term energy injection (Perna et al. 2006; Kumar et al. 2008; Geng et al. 2013 and many references therein).

Discriminating between these two models to explain the continued activity of the central engine is beyond the scope of this work. However, the pulsar model could be further explored by a comparison between the pulsar rotational energy and the GRB energy and the similarities between the pulsar environment and the circumburst medium (e.g., Dai \& Lu 1998), with the aid of regular observational campaigns. The black hole hyperaccretion model can be further investigated by searching for observational signatures of an accretion disc (e.g., Perna et al. 2006; Cannizzo et al. 2011).

In the case of GRB 100418a, the continued injection of energy into the forward shock can also explain the long-term radio emission. The forward shock may have been continuously fed with energy from the activity of the central engine, heating up the gas in the surrounding medium and accelerating electrons to relativistic velocities in an optically thick region. Therefore, the afterglow kept rising until it reached its peak and lasted much longer than many other GRBs. With the expansion and deceleration of the emitting sphere, the radio afterglow eventually started fading slowly. In addition to the observed results, a simple mathematical formulation of the standard blastwave model for GRB afterglows (Granot et al. 2003; Taylor et al. 2004; Pihlström et al. 2007) was used to predict the theoretical upper limit for angular size of GRB 100418a, based on a standard cosmology and on the day it was observed with the VLBA, <0.04 mas. This upper limit is much smaller than the upper limit estimated using the data from the observations $(<0.33$ mas) but is consistent with the conclusion that the afterglow was unresolved even more than 60 days after the prompt emission, indicating that the forward shock or the shells of material were not expanding fast enough (i.e., slow-moving ejecta) that it could be resolved.

Since both observational and theoretical estimations of the limits on the size and speed of the afterglow lead us to the conclusion that the range of Lorentz factors model is only partially valid, it is likely that the long-term activity of the central engine is required to explain the longevity of the afterglow.

The slow rate of expansion of the afterglow implies that the material the ejecta were interacting with was dense, consistent with the persistent and strong radio emission. But due to the faintness of the GRB 100418a host galaxy (e.g., from Sloan Digital Sky Survey images; Malesani 2010), it is not possible to determine whether or not the GRB occurred in a high-density region (close to the center) of the galaxy.

\section{CONCLUSIONS}

GRB 100418a was an unusual GRB in terms of the duration and properties of its afterglow. The afterglow light curves showed a rare "plateau phase" that is thought to be a signature of the physical process associated with the longevity of the afterglow. A long-term observational campaign was carried out with the VLA, ATCA, and VLBA to monitor the afterglow in the radio band. The behavior of the ATCA + VLA light curve shows that the radio afterglow started to rise after a brief period of decline, indicating some kind of energy injection that re-energized the forward shock, which in turn produced a longlasting afterglow.

We used our radio observations to test one of the postulates of the energy injection model, deriving constraints on the outflow Lorentz factor and the GRB afterglow mass. It was found that the upper limits only partially support the notion that a range of Lorentz factors might have continuously supplied energy to the forward shock to produce long-lasting afterglow and that it could only be true for slow-moving shells with Lorentz factors $\gamma<7$. Therefore, in the case of GRB 100418a, it is inferred that it was only the slow-moving shells that could have contributed to the continuous energy injection to the forward shock at late times. There must have been significant contribution to the long-lasting afterglow from some other mechanism, e.g., the continued activity of the central engine, from which the forward shock continued to obtain sufficient energy to accelerate particles from the beginning and the slow-moving shells caught up with it at late times. Our GRB 100418a monitoring suggests that it is very important to keep searching for and not to miss a GRB afterglow as unusual and long-lasting as GRB 100418a, which should then be continuously monitored with frequent sampling in order to further test GRB theories.

This work made use of the ATNF CASS' ATCA and the NRAO's VLA and VLBA. We thank the schedulers and all of the support staff at NRAO and ATNF CASS. The Australia Telescope Compact Array is part of the Australia Telescope National Facility, which is funded by the Commonwealth of Australia for operation as a National Facility managed by CSIRO. The National Radio Astronomy Observatory is a facility of the National Science Foundation operated under cooperative agreement by Associated Universities, Inc. The research was 
supported in part by the National Natural Science Foundation of China (NSFC) under No.11073042, National Basic Research Program of China (973 Project 2009CB824800). Z.W. is a Research Fellow of the One-Hundred-Talents project of the Chinese Academy of Sciences.

\section{REFERENCES}

Antonelli, L. A., Maund, J. R., Palazzi, E., et al. 2010, GCN Circ, 10620 (http://gcn. gsfc.nasa.gov/gcn3/10620.gcn3)

Cannizzo, J. K., Troja, E., \& Gehrels, N. 2011, ApJ, 734, 35

Chandra, P., \& Frail, D. A. 2010, GCN Circ, 10650 (http://gcn.gsfc.nasa.gov/ gcn3/10650.gcn3)

Chandra, P., \& Frail, D. A. 2012, ApJ, 746, 156

Cucchiara, A., \& Fox, D. B. 2010, GCN Circ, 10624 (http://gcn.gsfc.nasa.gov/ $\operatorname{gcn} 3 / 10624 . g \mathrm{cn} 3)$

Dai, Z. G., \& Lu, T. 1998, A\&A, 333, L87

Deller, A. T., Brisken, W. F., Phillips, C. J., et al. 2011, PASP, 123, 275

Deller, A. T., Tingay, S. J., Bailes, M., \& West, C. 2007, PASP, 119, 318

Dermer, C. D. 2004, ApJ, 614, 284

de Ugarte Postigo, A., Thöne, C. C., Goldoni, P., et al. 2011, AN, 332, 297

Duncan, R. C., \& Thompson, C. 1992, ApJL, 392, L9

Frail, D. A., Berger, E., Galama, T., et al. 2000, ApJL, 538, L129

Frail, D. A., Kulkarni, S. R., Nicastro, L., Feroci, M., \& Taylor, G. B. 1997, Natur, 389, 261

Geng, J. J., Wu, X.-F., Huang, Y.-F., \& Yu, Y.-B. 2013, arXiv:1307.4517

Granot, J., Nakar, E., \& Piran, T. 2003, Natur, 426, 138

Greisen, E. W. 2003, ASSL, 285, 109

Grupe, D., Gronwall, C., Wang, X.-Y., et al. 2007, ApJ, 662, 443

Jia, L. W., Wu, X.-F., Lü, H.-J., Hou, S.-J., \& Liang, E.-W. 2012, RAA, 12, 411

Katz, J. I. 1994, ApJ, 422, 248

Katz, J. I., \& Piran, T. 1997, ApJ, 501, 425

Klose, S. 2010, GCN Circ, 10616 (http://gcn.gsfc.nasa.gov/gcn3/10616.gcn3)

Kulkarni, S. R., Djorgovski, S. G., Ramaprakash, A. N., et al. 1998, Natur, 393, 35

Kumar, P., Narayan, R., \& Johnson, J. L. 2008, Sci, 321, 376

Liang, E.-W., Zhang, B., O'Brien, P. T., et al. 2006, ApJ, 646, 351

MacFadyen, A. I., \& Woosley, S. E. 1999, ApJ, 524, 262

MacFadyen, A. I., Woosley, S. E., \& Heger, A. 2001, ApJ, 550, 410

Malesani, D. 2010, GCN Circ., 10621 (http://gcn.gsfc.nasa.gov/gcn3/ 10621.gcn3)
Marshall, F. E., Antonelli, L. A., Burrows, D. N., et al. 2011, ApJ, 727, 132

Meszaros, P., \& Rees, M. J. 1993, ApJ, 405, 278

Meszaros, P., \& Rees, M. J. 1997a, ApJ, 476, 232

Meszaros, P., \& Rees, M. J. 1997b, ApJL, 482, L29

Metzger, B. D., Giannios, D., Thompson, T. A., Bucciantini, N., \& Quataert, E. 2011, MNRAS, 413, 2031

Moin, A. 2012, PhD thesis, Curtin Univ. (http://espace.library.curtin.edu.au:80/ $\mathrm{R} ?$ func $=$ dbin-jump-full\&local_base $=$ gen01-era02\&object_id=187112)

Moin, A., Tingay, S., Phillips, C., et al. 2010, GCN Circ, 10832 (http://gcn.gsfc.nasa. gov/gcn3/10832.gcn3)

Niino, Y., Hashimoto, T., Aoki, K., et al. 2012, PASJ, 64, 115

Panaitescu, A., \& Kumar, P. 2002, ApJ, 571, 779

Panaitescu, A., Meszaros, P., \& Rees, M. J. 1998, ApJ, 503, 314

Perna, R., Armitage, P. J., \& Zhang, B. 2006, ApJL, 636, L29

Pihlström, Y. M., Taylor, G. B., Granot, J., \& Doeleman, S. 2007, ApJ, 664, 411

Pradel, N., Charlot, P., \& Lestrade, J. F. 2006, A\&A, 452, 1099

Raine, D. J., \& Thomas, E. G. 2001, in An Introduction to the Science of Cosmology, XII (Bristol: Institute of Physics Publishing), 220

Rees, M. J., \& Meszaros, P. 1992, MNRAS, 258, 4

Rees, M. J., \& Meszaros, P. 1994, ApJL, 430, L93

Rees, M. J., \& Meszaros, P. 1998, ApJL, 496, L1

Sari, R., Piran, T., \& Narayan, R. 1998, ApJL, 497, L17

Sault, R. J., Teuben, P. J., \& Wright, M. C. H. 1995, in ASP Conf. Ser. 77, Astronomical Data Analysis Software and Systems IV, ed. R. A. Shaw, H. E. Payne, \& J. J. E. Hayes (San Francisco, CA: ASP), 433

Shepherd, M. C. 1997, in ASP Conf. Ser. 125, Astronomical Data Analysis Software and Systems VI, ed. G. Hunt \& H. E. Payne (San Francisco, CA: ASP), 77

Tagliaferri, G., Goad, M., Chincarini, G., et al. 2005, Natur, 436, 985

Taylor, G. B., Carilli, C. L., \& Perley, R. A. (ed.) 1999, in ASP Conf. Ser., Synthesis Imaging in Radio Astronomy II (San Francisco, CA: ASP)

Taylor, G. B., Frail, D. A., Berger, E., \& Kulkarni, S. R. 2004, ApJL, 609, L1

Taylor, G. B., Momjian, E., Pihlström, Y., Ghosh, T., \& Salter, C. 2005, ApJ, 622,986

Thompson, C. 1994, MNRAS, 270, 480

Waxman, E. 1997a, ApJL, 485, L5

Waxman, E. 1997b, ApJL, 489, L33

Waxman, E. 1997c, ApJL, 491, L19

Waxman, E., Kulkarni, S. R., \& Frail, D. A. 1998, ApJ, 497, 288

Wijers, R. A. M. J., Rees, M. J., \& Meszaros, P. 1997, MNRAS, 288, L51

Wilson, W., Ferris, R. H., Axtens, P., et al. 2011, MNRAS, 416, 832

Zhang, B., \& Meszaros, P. 2001, ApJL, 552, L35

Zhang, B., Fan, Y. Z., Dyks, J., et al. 2006, ApJ, 642, 354 